\title{
2018 WSGC Elijah High Altitude Balloon Final Report
}

\author{
Quin Bock', Tim Buchmann², Soren Zhane Chapman-Aldridge², Jake Dregne², Nicole Jackson², \\ Cody Nettesheim ${ }^{2}$ \\ 'Lawrence University, ${ }^{2}$ Milwaukee School of Engineering
}

\begin{abstract}
The 2018 Elijah Payload Project focused on designing, fabricating, and flying a high-altitude balloon payload. This payload had six different research topics: 360 degree video, atmospheric composition, flight path predictors, radiation sensors, sonification, and lighting fire at high altitudes. Flight predictors were run before and during the flight, and the payload included a separate GPS tracking payload to give us its exact location.
\end{abstract}

\section{Introduction}

The 2018 Elijah Payload Project is a ten-week project where a group of six students from STEM backgrounds design and build a balloon to be launched on a high-altitude weather balloon near the end of the project. The payload must be under six pounds and designed to be destructible, in case of a crash. The students must design, build, and fly their payload, and then recover and analyze the data collected. The main goal of the project being to design new experiments and continue work on experiments from past team while improving engineering, communication, and team skills.

\section{Payload Construction}

The payload was required to meet specific qualifications regarding weight and pressure. These had to be taken into account when designing and constructing the payload. In order to fit within FAA regulations our payload needed to be a maximum of six pounds and no one surface could have more than three pounds per square inch of impact force. Due to this we decided to go with a cylindrical design. It allowed all the surfaces to be large enough to not have too much of an impact force and is easy to build.

The housing for all of our parts and components was made from hard pink foam insulation. The foam is a great insulator from heat loss due to conduction, as well as being a sturdy material that is easy to work with and assemble with a 3M Styrofoam spray adhesive. Using a hot wire two circles and six rings were cut out. These were they glued together to form the entire housing for the interior which held all of the electronics. Once the housing was glued together it was covered in a layer of mylar to prevent heat loss due to radiation.

Within that housing was a body tube with 3D printed plate holders glued into it. The body tube was designed to allow easy access to the components. One could simply slide the tube in and out of the foam housing to gain access. The holders were at arc of 135 degrees and had a slit which the plates could slide into and fit snug. The payload design worked well. All fit inside well and held tight together to prevent contamination or loss of any parts. Everything was intact upon landing and safe and secure inside. 


\section{Control Systems}

The control system consists of all the electronic components that record information and control the experiments on board the payload. The purpose of the controls position is to make certain that each team's subsystem is operating correctly, and integrated properly into the payloads computer system. This required tracking each team's progress throughout their development and the communication standards they were using. This was to make sure that our microcontroller's IO Ports could handle each communication protocol, and the amount of sensors required. The payloads primary functions are controlled by an Arduino Mega 2560. The majority of the sensors on the payload are on an I2C network, which makes connection simple, only requiring two wires to connect the sensors to the arduino (not including each sensors power). Since we have several I2C components we needed to build an I2C Pullup Resistor network for the I2C transmission lines (Data, Clock) to work properly as shown in figure 1.

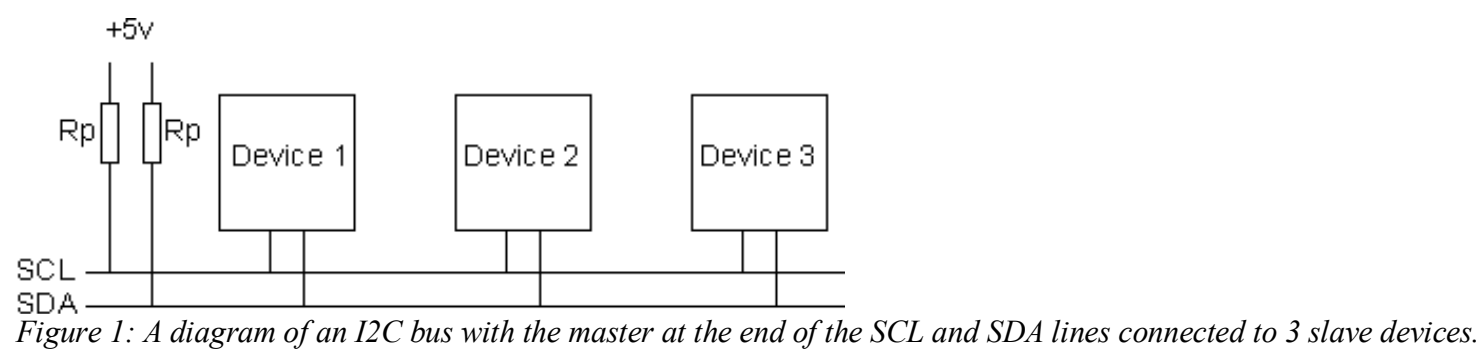

Doing this alters the relative voltage between the slaves to work at the appropriate low and high values, which ensures that the I2C Start and Stop sequences are properly received and transmitted.

\section{Power}

Every project needs power to operate, and this project is no exception. To calculate the power that would be necessary we made a table of all sensors and devices that would draw power. Most objects in our design run continuously, drawing a constant current and voltage, making it very easy to estimate the power absorbed. When we know how much power we need to provide, we can decide how many batteries we will need.

Before we started wiring the payload, we decided on a wire color code. We decided that wires carrying ground level voltage would be black, $3.3 \mathrm{~V}$ would be yellow, and $5 \mathrm{~V}$ would be red. We also decided that it would work best to have 2 separate circuits within the payload. Our first circuit would have most of our devices, and everything would run continuously. This circuit would draw just short of a watt. Our second circuit would just be for testing our space fire experiment. This way that experiment, if gone wrong could not short or wreck the other circuit elements.

\begin{tabular}{|l|l|}
\hline $\mathbf{1}$ & Batteries \\
\hline $\mathbf{2}$ & Battery Holders \\
\hline $\mathbf{3}$ & Variety of Wire Colors \\
\hline
\end{tabular}

Our flight was a success and we had plenty of power for our flight. Even though the flight went 3 hours longer than the expected flight time the payload still had power when we recovered it.

Figure 2: A list of materials for the control system 


\section{Flight Path Analysis}

In the past the Elijah Balloon team has only used one high altitude balloon prediction model to predict the paths of their balloon. This year as one of our projects we wanted to look into other flight prediction softwares and how they performed against each other. To do this we created a program that took the csv files from all the predictions and the actual flight as inputs and compared the deviations between the paths giving us a list of deviations along the flight path, average deviation, and standard deviations of all these deviations. We decided to test the veteran Cambridge prediction software that the Elijah Balloon project has been using for years, the ASTRA predictor from University of Southampton, University of Wyoming's predictor, and predictor software from S3research. To test all of these prediction softwares we produced predictions of our flight and then took the data from the actual flight and compared each prediction to the actual flight data in our program. The prediction with the lowest average deviation and tightest standard deviation would be the best prediction.

We decided to calculate the deviations between data points with the closest unix timestamp by calculating the straight line distance between the two points. Once all of these deviations were calculated then an average deviation was derived as well as a standard deviation of the deviations. This gave us a measure of how close all the deviations were to the average deviation. This was a relatively simple task which we programed in $\mathrm{C}++$ as well as functions to read in and parse the csv files from the predictions and actual flight paths. The full code, and prediction files are available on my GitHub: https:/github.com/qbock/WSGC-Elijah-Balloon-Flight-PathComparison.

\begin{tabular}{|c|c|c|c|c|c|c|c|c|}
\hline $\begin{array}{l}\text { Actual } \\
\text { Timestamp }\end{array}$ & Actual Latitude & Actual Longitude & Actual Altitude & $\begin{array}{l}\text { Prediction } \\
\text { Timestamp }\end{array}$ & Prediction Latitude & $\begin{array}{l}\text { Prediction } \\
\text { Longitude }\end{array}$ & Actual Altitude & $\begin{array}{l}\text { Deviation } \\
\text { (Degrees) }\end{array}$ \\
\hline 0 & 42.9133 & -89.19793333 & 882.546 & 0 & 42.9116 & -89.1979 & 267 & 0.001700327 \\
\hline 480 & 42.91328667 & -89.19788 & 912.074 & 480 & 42.90245 & -89.18905 & 932 & 0.013978638 \\
\hline 919 & 42.90970667 & -89.20257333 & 2306.431 & 919 & 42.8894 & -89.1795 & 1527 & 0.030736613 \\
\hline 1399 & 42.89861333 & -89.20568 & 4560.368 & 1399 & 42.865752 & -89.155856 & 2225.6 & 0.05968499 \\
\hline 1793 & 42.87828 & -89.19990667 & 5892.389 & 1793 & 42.84428571 & -89.13155714 & 2777 & 0.076336553 \\
\hline 2273 & 42.86866 & -89.18829333 & 7513.124 & 2273 & 42.8196 & -89.1011 & 3417 & 0.100047791 \\
\hline 2716 & 42.84130667 & -89.16750667 & 9271.654 & 2716 & 42.7962 & -89.0674 & 4047 & 0.109799622 \\
\hline 3196 & 42.818686667 & -89.15137333 & 10731.628 & 3196 & 42.76844167 & -89.02488333 & 4741.166667 & 0.13610393 \\
\hline 3214 & 42.81735333 & -89.15001333 & 10833.334 & 3214 & 42.7682 & -89.0245 & 4747 & 0.134794829 \\
\hline 3274 & 42.81618 & -89.14858667 & 10928.478 & 3274 & 42.7652 & -89.0198 & 4817 & 0.138509808 \\
\hline
\end{tabular}

Table 1: The first 10 results for the flight path prediction comparing software.

Unfortunately, due to the fact the we didn't have enough helium to get the five extra pounds of lift we don't have accurate predictions for many of the softwares we were testing. This five extra pounds would have yielded a lift rate of $5 \mathrm{~m} / \mathrm{s}$ instead our rise during the flight of approximately 
$1.4 \mathrm{~m} / \mathrm{s}$. All of the original predictions were based on the 5 extra pounds of lift and $5 \mathrm{~m} / \mathrm{s}$ rise rate. The only prediction tool that we were able to re-run predictions with accurate rise rates was the Cambridge predictor; even that wasn't perfect because the prediction tools don't allow you to make predictions with launch times earlier than the current time. This led to slightly different inputs for the weather data. Due to all of these complications and our ability to only carry out one flight, no clear distinction can be made between all of the prediction models. However what came out of this project was the creation of a tool that allows you to compare the "closeness" of flight paths.

\section{Balloon Flight}

A successful flight consists of launching our payload, recording our data, and recovering it. To do this there are a myriad of things that must be planned: the payload, tracking devices, and balloon must be connected properly, the flight path of the balloon shouldn't go through any major airspaces, the balloon must land in an accessible area, and we must keep track of where the balloon is going throughout the flight.

Approximately a week prior to the flight the team ran prediction models on various launch locations all over Wisconsin and surrounding states and look at the generated flight paths to see where they predict the balloon should land. It is important that we choose a place to launch from that will yield a flight path that stays clear of no-fly zones around cities and airports, and that will land in an accessible area such as an open field. We continue to run these predictions as we move closer to the day we fly as the models closer to the launch gives us more accurate predictions. To ensure the greatest confidence in the prediction models our final launch location determined the night before or the morning of launch.

Sometime in the week before the flight we carry out a mock flight so that when it comes time for the flight we are well versed in the procedure of launching the balloon and ready for any unanticipated events. When we arrive at our chosen launch site the day of the launch we lay out a tarp so that we save a surface to work on where we can see everything and nothing will get lost. First we weigh everything that will be in the balloon train and fill a bucket with gravel to match that weight plus 5 pounds for extra lift. This bucket is be attached to the balloon throat to indicate when the balloon is filled. When the bucket lifts completely off the ground we know we have enough lift. After the balloon is inflated we can complete the assembly of the balloon train which consists of the parachute, tracking payload, balloon, and lines between them. Then we are ready to launch our balloon; we release our balloon first, then slowly let go of each proceeding object in the payload train and then watch our balloon float away.

Before we can stare at the balloon too long we have to take off to chase and track the balloon so we can be there when and wherever it lands. This year we had three chase vehicles in which we followed the balloon. We did this using the location information packets from the new StratoStar tracking system and a secondary spot tracker combined with the predictions made prior to the flight to decide where to drive so that we could stay approximately under the balloon until its landing.

After a rough start and what we thought would be an unrecoverable payload we had a successful but lengthy flight. Our first predictions showed a nice flight path free of any major no fly zones, 
and landing in an area with a lot of agricultural fields. Due to the fact that we used up all of our helium in our second tank before we had our desired lift meant that instead of the balloon rising at an average calculated rate of $5 \mathrm{~m} / \mathrm{s}$ it averaged approximately $1.4 \mathrm{~m} / \mathrm{s}$. We then had to re-run predictions with the new rise rate, and these predictions revealed a flight path the landed the balloon close to the Chicago/Evanston area. Lucky the balloon didn't actually land this far east and hooked farther west landing us in a freshly cut grazing cattle pasture that belonged to a very friendly and helpful landowner. The payload was recovered with no damage and all of the data from the experiments intact and ready to be analyzed.

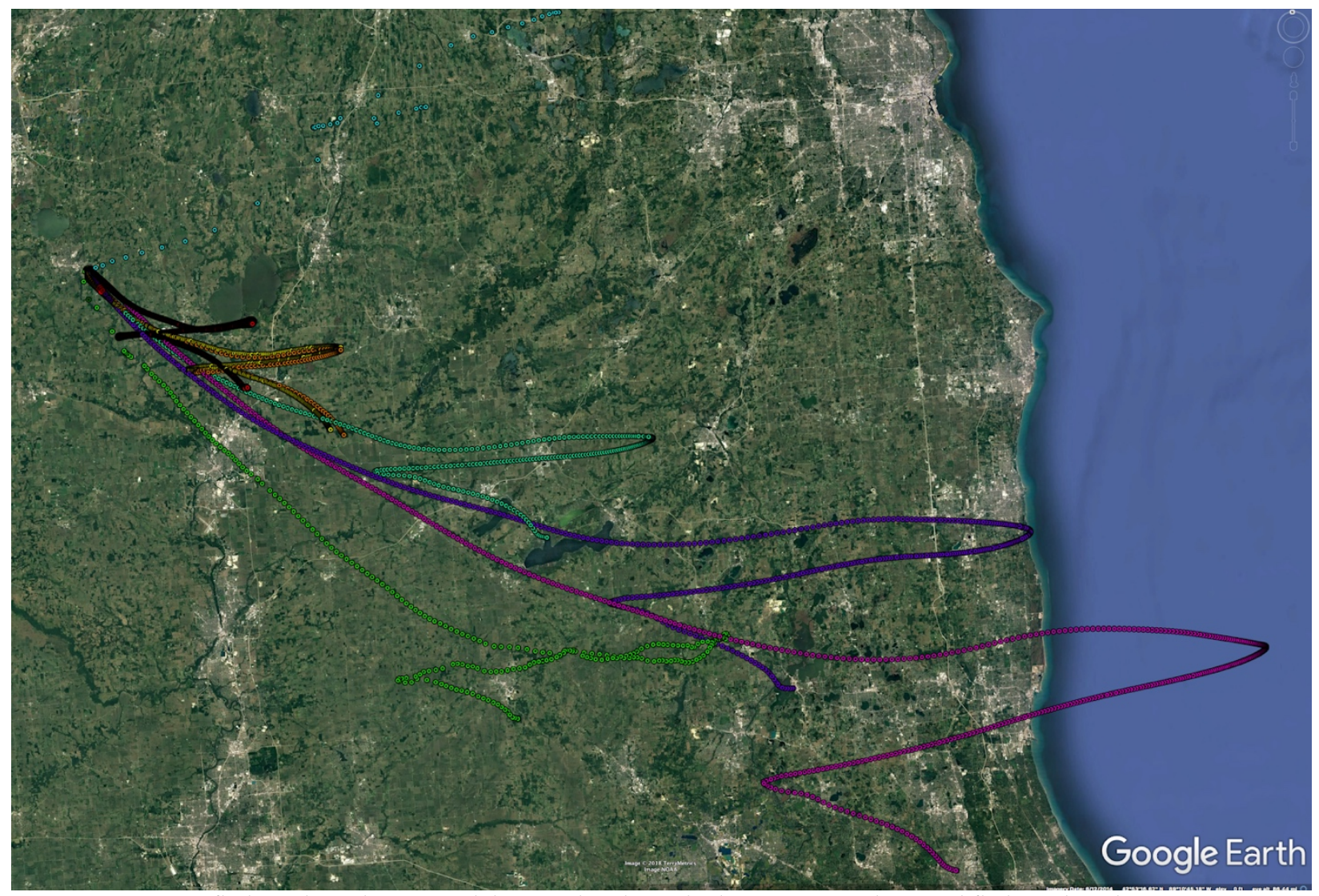

Figure 3 - Various flight path predictions and the actual path overlaid on one map. The actual flight path is shown in green, ASTRA predictor in red, Cambridge prediction ( $5 \mathrm{~m} / \mathrm{s}$ assent rate) in orange, Cambridge prediction (1.2 $\mathrm{m} / \mathrm{s}$ assent rate) in pink, Cambridge prediction (1.4 m/s assent rate) in purple, Cambridge prediction $(2.25 \mathrm{~m} / \mathrm{s}$ assent rate) in teal, $S 3$ research prediction in yellow, and the UWYO prediction in light blue.

\section{7. $360^{\circ}$ Video}

The goal of the $360^{\circ}$ video experiment is to fully capture the entire environment surrounding the payload as it ascends and descends in a $360^{\circ}$ virtual reality style video, as well as to investigate the feasibility of stabilizing such a video for easier viewing. The video was intended to be combined with the audio from the sonification experiment to create a fully immersive highaltitude experience.

Initially, several different methods of producing a $360^{\circ}$ virtual reality video were investigated. This includes dedicated $360^{\circ}$ cameras, traditional cameras with specialized lenses or concave mirrors, or an array of many traditional cameras stitched into a single video. Ultimately, it was 
decided that using 2 dedicated $360^{\circ}$ cameras would be easiest to implement with seamless results. The Kodak PIXPRO SP360 camera was selected as it was low cost and easy work with without sacrificing much quality. Additionally, methods of stabilization were investigated to provide a smoother viewing experience. This includes both active and passive stabilization of the payload, as well as stabilization of the video itself via post processing software. It was decided that passive and software stabilization would be used to minimize weight and complexity. Unfortunately, however, neither were ultimately attempted due to complications and time constraints. As aforementioned, two $360^{\circ}$ cameras were used; one on the top of the payload, and one on the bottom. Each camera captured a hemispherical view of the world around it, and the two videos were stitched into one after the flight using specialized software downloaded from the manufacturer's website. The cameras ran on their own internal batteries independent from the rest of the payload and stored the videos on $32 \mathrm{gb}$ microSD cards. The cameras could record approximately 2.5 hours of video before the batteries ran out of charge; this was plenty of time based on our predicted flight time. The cameras were attached to $3 \mathrm{~d}$-printed mounts placed inside the payload body with the camera lenses protruding from holes cut into the top and bottom of the payload.

The cameras performed well throughout the flight, successfully capturing footage which was processed into a full $360^{\circ}$ virtual reality video of the flight. The audio of the sonified data was also integrated into this video. Unfortunately, there were some issues; the cameras could only capture 2.5 hours of video, but due to a shortage of helium, the balloon rose very slowly, resulting in a much longer flight than expected. As a result, much of the flight was not possible to capture on film, including the bursting of the balloon as well as the entirety of the descent. In addition, condensation accumulated on the center of each lens as the payload reached high altitude, causing part of the view to be blurred for much of the video. As previously mentioned, stabilization techniques were not implemented, making the beginning of the video difficult to view as the payload spun very rapidly after launch and took several minutes to stabilize on its own. Ultimately, however, the $360^{\circ}$ video experiment was an overall success, despite these issues that would need to be worked out if the project were to be further developed.

\section{Atmospheric Composition}

The different attributes of the atmosphere have huge effect on our everyday life; from the air we breathe to the weather to the solar radiation we experience. Our goal with this experiment is to test the atmosphere as we fly. After that we will analyze the data and make conclusions about the atmosphere at the time of flight.

First off, we had to decide what types of data we wanted to collect. We based our decisions on sensor shipping time, the complexity of the sensor, and the meaningfulness of the data it would collect. We chose most of our sensors from AdaFruit, because of their quick shipping time and online resources to help us wire and code. We also got sensors from SPEC Sensors, Maritex, Seeed, WaveShare, and SparkFun. As for what we wanted to sense, we decided on pressure, temperature, and humidity to provide a baseline for us to compare our data to; dust amount, carbon dioxide, and benzene to help tell the quality of the air; ozone and chlorofluorocarbon (CFCs) to measure the amount of ozone, as well as provide data on CFCs, a chemical that destroys ozone; and oxygen amount to provide more meaning to the space fire research project. 
The next step was to test each sensor individually to make sure that they would work. Two of our sensors use $\mathrm{I}^{2} \mathrm{C}$ data logging, but using libraries and instructions from AdaFruit we were able to get them to work. Our other sensors were analog read sensors, so were very easy to code and test. However, we couldn't get our dust sensor to work properly, even when we tested it with smoke, so we decided not to use it. Next, we had to make sure the sensors would work together. This was rather difficult due to the $\mathrm{I}^{2} \mathrm{C}$ setup of the sensors and obtaining proper baseline readings. After that, we had to program our data shield to print our data to an SD card. After we figured that out, we soldered our sensors to a breadboard, making sure to color code our wires.
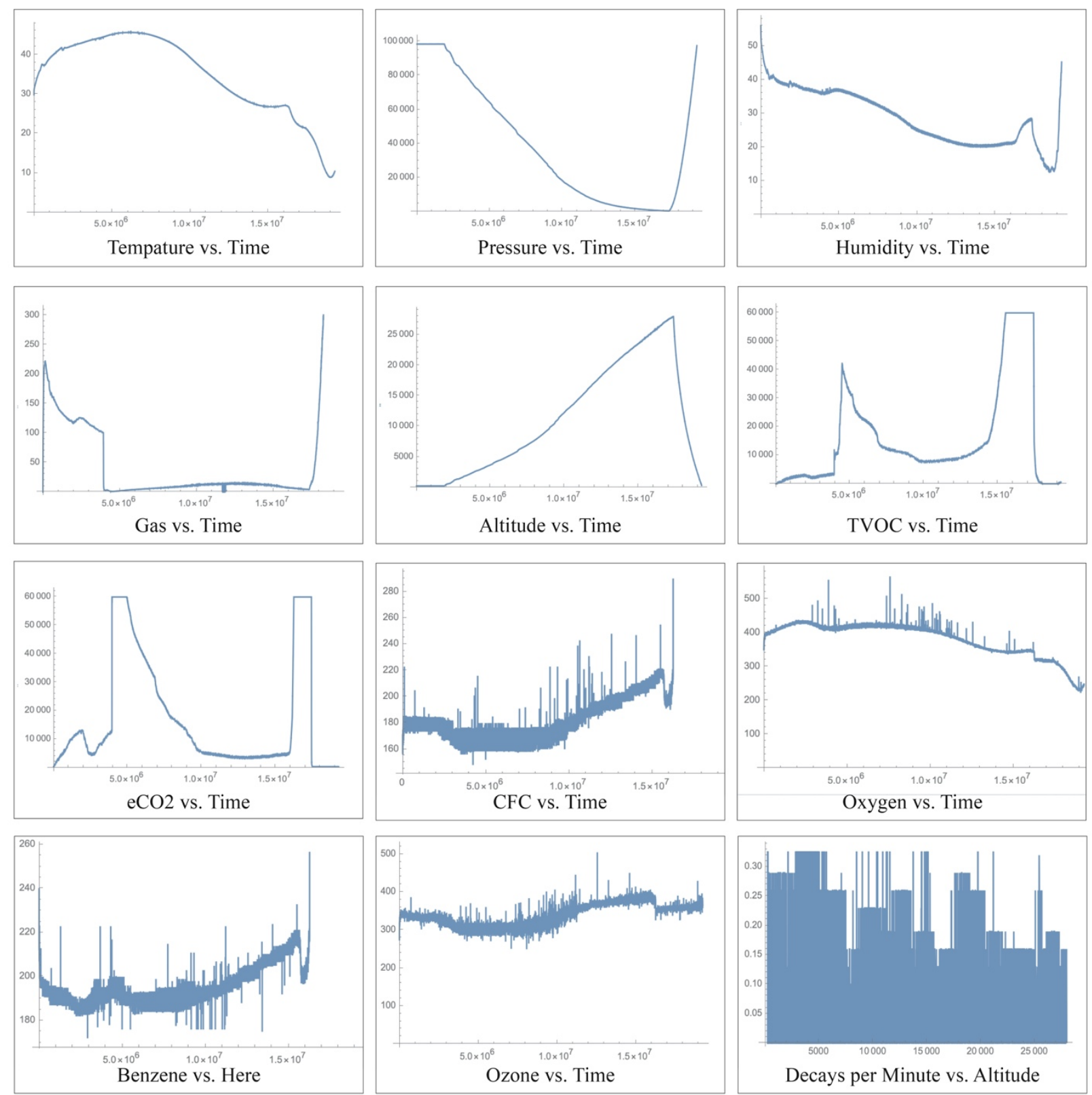

Figure 4: Graphs of various sensor measurements vs. time. 
These graphs display all the data we were collecting with our on-board sensors. From this we can immediately notice a few things. The altitude indicates that the balloon pops at about 17500 milliseconds. Also, indicated by a flat line at a value of 60000 some of the sensors maxed out their value, this indicates very high levels of that type of gas.

Each graph has different units, so it's hard to compare the reading levels to one another based solely on the values. Benzine, Ozone, equivalent calculated carbon-dioxide $\left(\mathrm{eCO}^{2}\right)$, and $\mathrm{CFC}$ data was recorded in parts per million, whereas the Total Volatile Organic Compounds is represented in parts per billion. The pressure readings are in pascals, and the altitude is in meters. The oxygen data is just a scaled percentage of how much oxygen is in the air, with a maximum value being 25\%. Different units makes it difficult to compare the values of each respective graph. However, the graphs still clearly show the changes in each of the represented gases as we rise in altitude and what is perhaps more interesting than the specific values of each gas at a specific altitude is the overall trends in the gas levels represented by the graph and how they relate to each other.

\section{Radiation}

The Radiation aspect of the project is to accurately measure exposure of three parts of the electromagnetic spectrum. The three we chose to monitor is ultraviolet, beta, and gamma rays. The original plan of the group was to use film to test for radiation levels, however, we found a more efficient and quantitative ways of measuring radiation.

To measure for Beta and Gamma rays the most reasonable way we found was to either purchase or build a Geiger Counter. When we looked at prebuilt geiger counters online, we found that they were pricey, large, and didn't suit our needs. We then came across kits to build a geiger counter, and kits specifically designed to work with Arduino products. We chose the Mighty Ohm Geiger Counter and used an Arduino Nano to store the data. We then selected a UV sensor from Adafruit to measure UV levels. The sensor then connected to the Arduino.

During the flight, we recorded Beta and Gamma levels that slowly rose with altitude. This was something we predicted. However, we realized shortly after launch that there was never an access hole cut for the UV sensor, so we did not get an accurate reading for UV levels.

\section{Sonification}

Sonification is the conversion of one type of data to sound. Two great examples of this are heart rate monitors, and radar pings. We decide to sonify our data as a means of scientific outreach. We wanted the audience to hear the environment changing as the flight footage was playing; it was our hope that this would provide a scientific wow factor, even for those who are not scientifically trained. The payload logged our data on to and SD card, and it was stored in a CSV file. When the data was imported in to the sonification software, it was exported as a MIDI file, which is an industry standard music file for sequencing notes, their lengths, and their frequencies.

The sonification program was written in Java utilizing mostly Java native libraries, only with the usage of two external libraries: OpenCSV and JMusic. OpenCSV provided a much more robust and user friendly interface for importing the CSV flight data than the basic Java CSV class. After 
importing, the flight data was then scaled down to a range of 0-127 to make it fit on the MIDI musical scale, this allowed for all of the data to be within audible range. Once this process was completed for each of our sensor's data, the newly manipulated data was exported to a MIDI file, which could then be modified using any external music editing software.

Once the MIDI file was exported from the sonification software, it was then imported into a free version of Ableton Live, where each sensor could be attributed its own sound. The result: an eerie and cacophonous orchestra of scientific data.

\section{Space Fire}

Fire is a very complicated substance that is both fascinating and dangerous. It needs heat to light, and then fuel and oxygen to stay lit. If you take away either one of those, the fire will go out. For this fire experiment, four fires were lit at different altitudes, some of which should not be able to support the fire.

The way the matches were lit was using a nichrome wire. Nichrome wire has a very high resistance and so when current is run through it heats up more quickly than other wires. This allowed us to use them as our ignition method we simply wrapped a the tip of a match in wire and ran a current through it until the match lit and then the current was shut off. The method here also had the added benefit of being very simple to control all that needed to be done was turn a pin on for power and then turn it off after a set amount of time. Relays were also used in order to better control the flow of current so there was not an accidental ignition. The system that was created in order to observe the matches lighting consisted of a spy camera and and infrared camera mounted to a holder we designed and 3D printed which attached to a servo. That then spun from match to match in order to observe them as they lit. The code we developed worked with the altimeter in order to begin the lighting of the matches as we reached certain heights we wanted to test at. The birch plate which it was mounted on was covered in aluminum foil in order to prevent it igniting although that would have been very difficult. The plates were a dense plywood that was hard to light. On the plate then was mounted the bank of relays which was used as well as small dividing walls between the four chambers for the matches. The small walls were also covered in aluminum foil just in case. Then the matches were held in place using paper clips that were swirled into holders around the stick which were stuck and glued into the plate.

During our flight all matches successfully were ignited. While recovering the payload it was discovered that all matches were ignited. Also as the payload was discovered it was noticed that the camera mount had been broken off and had tipped over. Due to that there is no actual footage of the flames. At times one can see light from a flame but other than that sadly no other observations can be made. Another flight with a better secured camera holder would be necessary in order to discover what actual heights the matches were ignited at and more details that can be observed.

\section{Conclusion}

From joining as a team in early June to launching and recovering our payload in August, the 2018 Elijah Balloon Project has been a success. In just ten weeks our six members and one advisor have joined as a team, learned roles, and gotten to know each other as colleagues and friends. On top of that in these ten weeks we went through the research of many ideas and 
experiments and narrowed them down to six that would be a part of our payload. In the remaining weeks we continued research on our respective projects and started developing them. Nearing the end of the ten weeks we had together we finally developed and built a payload that would fly into a near space environment. We also learned the logistics of launching high altitude balloons, and successfully launched and recovered our payload. We were happy to find that nearly everything on the payload worked as we expected. Due to the complication with our flight there wasn't much meaningful flight path data to analyze, however we did develop working flight path analysis software. We successfully recorded and stitched a full 360 degree video of most of the flight. Sonification produced an eerie, sci-fi sounding track based off our data to accompany the video. Although there was an unforeseen camera mount failure, the matches did ignite at their respective altitude, and we would have only needed a second flight to observe the flame. Finally, all of the sensors but the UV sensor recorded meaningful data, with interesting correlations. We were pleased with the success of our flight and our presentation on project day later at the WSGC Conference in Fox Valley. We are excited to provide helpful material for future Elijah High Altitude Balloon Teams.

\section{Acknowledgements}

Dr. William Farrow

Christine Bolz

Lisa Crumble

Richard Phillips

A special thanks to NASA and WSGC for continuing to provide this opportunity to us and students like us. 